\title{
Medicamentos peligrosos
}

\section{Hazardous drugs}

Pedro García Salom ${ }^{1}$ (1) 0000-0001-5235-4722

${ }^{1}$ Hospital General Universitario, Servicio de Farmacia, Alicante, España.

\section{Correspondencia}

Pedro García Salom

garcia.salom.pedro@gmail.com

Recibido: 13.01.2021

Aceptado: 17.01 .2021

Publicado: 31.03 .2021

\section{Financiación}

Sin financiación

\section{Conflicto de intereses}

No hay conflicto de intereses

\section{How to cite this paper}

García P. Medicamentos peligrosos. Med Segur Trab (Internet). 2021;67(262):6-10. doi: 10.4321/s0465$546 \times 2021000100001$

(a) BY-NC-SA 4.0 
Las propiedades tóxicas de los medicamentos citostáticos son bien conocidas desde que en los años 40 empezaron a utilizarse en el ámbito oncológico(1).

Sin embargo, hasta que en la década de los 80 se comenzaron a publicar una serie de estudios posteriores $^{(2,3)}$, cuyos resultados apuntaban a la posible relación entre la exposición laboral a citostáticos con el incremento de diferentes efectos para la salud, ni los organismos públicos ni las sociedades científicas habían tomado cartas en el asunto. Es a partir de este momento cuando empieza la concienciación del problema y surgen las primeras directrices y recomendaciones.

En 1981 aparece la primera guía para el manejo seguro de los medicamentos citostáticos $^{(3)}$ editado por la Sociedad Australiana de Farmacéuticos de Hospital y unos años más tarde se incorpora la Sociedad Americana de Farmacia Hospitalaria. En la década de los 90 se acuñó el término «medicamento peligroso» por la sociedad americana de farmacéuticos del sistema público americano ${ }^{(4)}$.

Sin embargo, no es hasta que en septiembre 2004 este término no es adoptado por la Instituto Nacional de Seguridad y Salud Ocupacional (NIOSH), cuando empieza a conocerse el problema de forma más generalizada y comienza a tomarse conciencia del problema en profundidad ${ }^{(5)}$.

Este organismo identificó una lista de las principales drogas peligrosas elaborada a partir de información proporcionada por otras cuatro instituciones que habían generado listas de medicamentos peligrosos para sus respectivas instituciones, así como una lista de Pharmaceutical Research and Manufacturers of America (PhRMA). La lista de 2004 se actualizó en 2010 y desde entonces está alcanzando una actualización bianual(6-10).

En estas monografías se establecen los criterios utilizados para ser incluido en las listas de medicamentos peligrosos y más recientemente, listados de medicamentos sospechosos de serlo también, que han sido evaluados pero que finalmente no alcanzan la toxicidad requerida, según criterios establecidos para ser considerados peligrosos en el entorno sanitario.

La difusión internacional de estos listados, ha evidenciado el retraso de los organismos españoles, que no habían desarrollado ningún marco de referencia hasta ese momento respecto del problema y que se vieron en la necesidad de tomar "cartas en el asunto" y adaptar estas listas a los medicamentos comercializados en España. Queda por dilucidar si serán capaces de poner en marcha criterios propios, o en su defecto, afrontar el reto de actualizar y adaptar para España estos listados de forma rápida y continuada, para evitar que las nuevas incorporaciones de estos medicamentos queden al margen de los procedimientos y prácticas de seguridad que ya se emplean de forma generalizada en España, o al menos establecer un procedimiento estándar detallado donde se recojan las fuentes de obligada consulta, en el entorno sanitario, para determinar inequívocamente su clasificación evitando así las discrepancias o disparidades de criterios entre comunidades autónomas o establecimientos sanitarios, antes de incorporarlos a la práctica asistencial.

Actualmente, podemos acceder a información de nuevos medicamentos incluidos por NIOSH cada dos años, publicados, en audiencia pública incluso como borradores previos antes de ser declarados oficiales por el organismo, la última la de 2020. Lamentablemente, no hemos visto una translación de esto en España. La base de datos del Instituto Nacional de Seguridad y Salud en el Trabajo (INSST) de medicamentos peligrosos (INFOMEP) puesta en marcha en 2016, no parece estar actualizándose de manera fluida y se encuentran medicamentos incluidos en actualizaciones de la NIOSH que a día de hoy no se recogen en esta base de datos de acceso público, si bien ya se recoge toda una declaración de intenciones de continuidad de información en dicha base de datos ${ }^{(11)}$.

Se ha asistido, en los últimos años en España, a un torbellino de medidas relacionadas con estos medicamentos, como la incorporación de equipos cerrados «Closed system transfer devices (CSTDs)» de alto coste para el sistema sanitario ${ }^{(12)}$ para aumentar la seguridad durante la manipulación, tal vez sin racionalizar suficientemente si los riesgos descritos que potencialmente reducen (menor cantidad de pérdidas y goteos, generación de aerosoles y formación de vapores), se contrarrestan al generar otros nuevos en comparación con los tradicionales sistemas de preparación.

Sirva como botón de muestra las pruebas realizadas sobre uno de estos dispositivos para su aprobación como sistema cerrado CSTDs, en los que fue necesario "forzar de manera artificial" la presión de 
vapor en los viales, para que el vapor generado fuese medible y poder demostrar que el sistema «TOXI-GUARD» (presente en ON-GUARD/TEVADAPTOR) es capaz de retener los vapores producidos, tal y como se recoge textualmente en la documentación del sistema: «La cantidad de vapores de fármacos que pueden generarse en condiciones normales de uso es extremadamente baja y, por lo general, está por debajo de los límites analíticos de detección». Paclitaxel, uno de los medicamentos ensayados, ni siquiera en estas condiciones extremas forzadas generó vapor suficiente para poder ser detectado incluso en los equipos desprovistos del sistema TOXI-GUARD de retención de vapor ${ }^{(13)}$.

Sin embargo, es importante saber que estos sistemas cerrados no están exentos de inconvenientes respecto a sistemas tradicionales. Es necesario ejercer con algunos de estos equipos una la fuerza extremadamente alta que para poder penetrar los tapones de los viales, provocando fatiga en los operarios, lesiones en las manos y rotura de los viales, así como, en ocasiones, la imposibilidad de extraer la totalidad del contenido del vial, generando a su vez una mayor cantidad de residuo de dicho medicamento y finalmente también está registrada una mayor frecuencia de desprendimiento de pequeños fragmentos del tapón que pueden ser extraídos junto al contenido de los viales tal y como se recogen en la base de datos MAUDE ${ }^{(14-15)}$.

La adecuación de la estructura física de los servicios de farmacia para su manipulación, con la construcción de salas blancas de presión negativa y utilización de cabinas de seguridad biológica con mayores tasas de extracción al exterior, está siendo otra de las grandes transformaciones de los servicios de farmacia, desde que en 2014 se publica la guía del ministerio de «buenas prácticas de preparación de medicamentos en servicios de farmacia hospitalaria» ${ }^{(16)}$ así como la utilización de un equipamiento de protección más adecuado y la implantación de procedimientos y prácticas seguras para minimizar la exposición de los trabajadores a estos medicamentos ${ }^{(17-19)}$.

Es posible pensar que con la implantación de estas medidas está cubierto en gran medida el reto de su utilización, pero nada más lejos de la realidad.

Se ha producido una pequeña revolución tecnológica orientada fundamentalmente a la elaboración de medicamentos peligrosos estériles, probablemente porque los citostáticos se han comercializado tradicionalmente y mayoritariamente por vía parenteral, pero mantenemos un vacío tecnológico o de NO regulación, en la manipulación de los medicamentos peligrosos no estériles. No debemos olvidar que los medicamentos y los productos químicos están regulados por normativas distintas, pero existen numerosos productos químicos peligrosos, que son utilizados por los servicios de farmacia para la elaboración de medicamentos, incorporándose a la cadena terapéutica por la "puerta de atrás" y escapando a la regulación de los medicamentos peligrosos.

Los organismos reguladores no han abordado el formato en que los laboratorios deberían comercializar los medicamentos líquidos peligrosos multidosis (ej. Jarabes, soluciones), teniendo que dosificarlos aun hoy día abriendo el frasco en su totalidad y dosificarlo con cucharadas, en domicilios por parte de familiares o del personal sanitario en hospitales, con el inevitable goteo, caídas de líquido, etc. En ocasiones y en el mejor de los casos, los frascos van provistos con tapones obturadores que requieren la utilización de jeringas dosificadoras para la extracción de las dosis, aunque este sistema tampoco resulta seguro, sí que reduce el contacto al manipular, pero no resuelve el problema satisfactoriamente.

Pero, sin duda alguna, el mayor reto que se tiene por delante, es el de la evaluación de los riesgos al manipular estos medicamentos.

Sabemos que hay multitud de elementos de minoración (instalaciones, equipos de protección individual, procedimientos, etc.) que pueden implementarse para reducir la exposición a los medicamentos peligrosos, durante los procesos de almacenamiento, preparación, transporte, administración, eliminación de residuos, cuidado de los pacientes, etc. Nadie duda que, en mayor o menor medida su implementación serviría para reducir la exposición a estos medicamentos ${ }^{(20,21)}$. Pero, se desconoce realmente cual es el impacto que cada uno de ellos puede aportar, sobre la minoración global que el conjunto de medidas conocidas podría conseguir, siendo necesario desarrollar una evaluación de riesgos metodológica adecuada, para abordar esta cuestión. 
Estamos desprovistos de registros a nivel nacional que recojan y documenten los incidentes y la frecuencia con la que estos se producen, identifiquen a los trabajadores expuestos, y las consecuencias futuras a largo plazo, que una mayor o menor exposición puedan tener. Deberíamos ser capaces de ponderar de forma plausible cual es el impacto que cada uno de los elementos de minoración tiene, sobre la reducción de la exposición, y esto no se puede realizar sin un registro prospectivo de incidentes, que puedan ser investigados teniendo en cuenta la actividad que se estaba realizando, los equipos utilizados, las circunstancias en las que se han producido, y los elementos conocidos de minoración sin implementar que pudieran haber contribuido a que se produjeran. La comparación con la frecuencia de otras instituciones, en las que se hayan implementado algunas de estas minoraciones, nos permitirá establecer un orden de prioridad de las mismas y planificar su incorporación futura, atendiendo a complejidad o los recursos necesarios para su incorporación.

El objetivo final no debe ser otro que poder establecer a partir de estos conocimientos, entornos de trabajo saludables para los trabajadores, en donde se exijan por normativa, la implantación de un determinado conjunto de elementos de minoración, que aseguren un grado adecuado de seguridad, para poder seguir realizando esta actividad, o que sirva incluso para acreditar a los nuevos servicios y establecimientos sanitarios que deban trabajar con estos medicamentos.

\section{Bibliografía}

1. Goodman LS, Wintrobe MM, Dameshek W, Goodman MJ, Gilman A, McLennan MT. Nitrogen mustard therapy; use of methyl-bis (beta-chloroethyl) amine hydrochloride and tris (beta-chloroethyl) amine hydrochloride for Hodgkin's disease, lymphosarcoma, leukemia and certain allied and miscellaneous disorders. J Am Med Assoc. 1946;132(3):126-32. doi:10.1001/jama.1946.02870380008004

2. Guidelines for safe handling of cytotoxic drugs in pharmacy departments and hospital wards. Hosp Pharm. 1981;16(1):17-20.

3. American Society of Hospital Pharmacists. ASHP technical assistance bulletin on handling cytotoxic drugs in hospitals. Am J Hosp Pharm. 1985;42(1):131-7.

4. American Society of Hospital Pharmacists. ASHP technical assistance bulletin on handling cytotoxic and hazardous drugs. Am J Hosp Pharm. 1990;47(5):1033-49.

5. Burroughs GE, Connor TH, McDiarmid MA, Mead KR, Power LA, Reed LD. NIOSH Alert: preventing occupational exposure to antineoplastic and other hazardous drugs in health care settings. Atlanta, USA: National Institute of Occupational Safety and Health (NIOSH), Department of Health and Human Services, Center for Disease Control and Prevention; 2004. Report No.: 2004-165.

6. Connor TH, Mackenzie BA, DeBord DG, Trout DB, O'Callaghan JP. NIOSH List of antineoplastic and other hazardous drugs in healthcare settings, 2014. Atlanta, USA: National Institute of Occupational Safety and Health (NIOSH), Department of Health and Human Services, Center for Disease Control and Prevention; 2014. Report No.: 2014-138.

7. Erce A, editor. Preventing occupational exposure to cytotoxic and other hazardous drugs: European Policy Recommendations. Brussels, Belgium: Rodhe Public Policy; 2016.

8. Braun B, Riehle A, Donofrio K, Hazif H, Loeb JM. Improving patient and worker safety: Opportunities for synergy, collaboration and innovation. Oakbrook Terrace, Illinois USA: The Joint Commission; 2012.

9. Occupational and Safety and Health Administration (OSHA). Controlling Occupational Exposure to Hazardous Drugs [Internet]. Washington DC, USA: OSHA, Department of Labor; 2016 [citado 24 de agosto de 2021]. Disponible en: https://bit.ly/39PeWNd

10. Pan American Health Organization - World Health Organization (PAHO/WHO). Safe handling of hazardous chemotherapy drugs in limited-resource settings [Internet]. Washington DC, USA: PAHO/WHO; 2013 [citado 24 de agosto de 2021]. Disponible en: https://bit.ly/2VvLQKe 
11. Instituto Nacional de Seguridad y Salud en el Trabajo (INSST). INFOMED -Información para trabajadores sanitarios sobre medicamentos peligrosos (Internet). Madrid, España: INSST [citado 24 de agosto de 2021]. Disponible en: https://bit.ly/3Dcln99

12. de Jong T, Pawlowska-Cyprysiak K, Hildt-Ciupińska K, Bos E, Nicolescu G, Trifu A, et al. Current and emerging occupational safety and health $(\mathrm{OSH})$ issues in the healthcare sector, including home and community care: European Risk Observatory Report. Luxembourg: European Union Publications Office; 2015.

13. Prevention of Hazardous Drug Vapor Release by the Tevadaptor ${ }^{\circ}$ Vial Adaptor (Internet). Bethlehem, USA: B Braun Medical Inc; 2014 [citado 24 de agosto de 2021]. Disponible en: https://bit.ly/38b4kpA

14. MAUDE - Manufacturer and User Facility Device Experience (Internet). Silver Spring, USA: U.S. Food and Drug Administration; 2021 [citado 24 de agosto de 2021]. Disponible en: https://bit.ly/3gsOJ9x

15. Zhao C, Radwick A. 5 Challenges of Closed System Transfer Devices (Internet). Bethesda, USA: Parenteral Drug Association; 2020 [citado 24 de agosto de 2021]. Disponible en: https://bit.ly/38bcSNb

16. Casaus Lara ME, coordinadora. Guía de buenas prácticas de preparación de medicamentos en servicios de farmacia hospitalaria (Internet). Madrid, España: Ministerio de Sanidad, Servicios Sociales e Igualdad; 2014 [citado 24 de agosto de 2021]. Disponible en: https://bit.ly/3moQh8l

17. García Salom P, López Briz E, Moya Gil A, Ferriols Lisart F, Marco Garbayo JL, Vila Clérigues N, et al. Guía para la adaptación de las buenas prácticas en la preparación y manipulación de medicamentos en la Comunidad Valenciana - Instalaciones (Internet). Valencia, España: Generalitat Valenciana; [citado 24 de agosto de 2021]. Disponible en: https://bit.ly/3kiOYVF

18. García Salom P, López Briz E, Moya Gil A, Ferriols Lisart F, Marco Garbayo JL, Vila Clérigues N, et al. Guía para la adaptación de las Buenas Practicas en la Preparación y manipulación de Medicamentos en la Comunidad Valenciana - Equipamiento (Internet). Valencia, España: Generalitat Valenciana; [citado 24 de agosto de 2021]. Disponible en: https://bit.ly/3mHdO4H

19. García Salom P, López Briz E, Moya Gil A, Ferriols Lisart F, Marco Garbayo JL, Gaspar Carreño M, et al. Guía para la adaptación de las buenas prácticas en la preparación y manipulación de medicamentos en los servicios de farmacia en la Comunidad Valenciana - Procedimientos (Internet). Valencia, España: Generalitat Valenciana; [citado 24 de agosto de 2021]. Disponible en: https://bit.ly/3DdRy87

20. USP $<800>$ Hazardous Drugs - Handling in Healthcare Settings Gap Analysis Survey (Internet). [citado 24 de agosto de 2021]. Disponible en: https://bit.ly/2WbXgH7

21. Hazardous Drug Consensus Group (HDGC). Consensus Statement on the Handling of Hazardous Drugs Per USP Chapter <800> [Internet]. Cary (NC), USA: Accreditation Commission for Health Care; 2017 [citado 24 de agosto de 2021]. Disponible en: https://bit.ly/37pzVTO 